\title{
Cooperative Compressive Power Spectrum Estimation
}

\author{
Dyonisius Dony Ariananda*, Daniel Romero ${ }^{\dagger}$, and Geert Leus* \\ *Faculty of EEMCS, Delft University of Technology, The Netherlands \\ ${ }^{\dagger}$ Dept. of Signal Theory and Communications, University of Vigo, Spain \\ \{d.a.dyonisius, g.j.t.leus\}@tudelft.nl and dromero@gts.uvigo.es.
}

\begin{abstract}
We examine power spectrum estimation from widesense stationary signals received at different wireless sensors. We organize multiple sensors into several groups, where each group estimates the temporal correlation only at particular lags, which are different from group to group. A fusion centre collects all the correlation estimates from different groups of sensors, and uses them to estimate the power spectrum. This reduces the required sampling rate per sensor. We further investigate the conditions required for the system matrix to have full column rank, which allows for a least-squares reconstruction method.
\end{abstract}

\section{INTRODUCTION}

The emergence of compressive sampling has renewed the interest in spectral analysis. The work in [1], for instance, focuses on signal reconstruction from sub-Nyquist rate samples produced by a multi-coset sampler. However, when only the statistics of the received signal are of interest (such as in a cognitive radio (CR) application), attempting to reconstruct the uncompressed waveform is unnecessary. In this case, power spectrum reconstruction from compressive measurements becomes more appropriate [2] [3]. Compressive power spectrum reconstruction for a wide-sense stationary (WSS) signal is possible, even for a non-sparse power spectrum, by exploiting the Toeplitz structure of its correlation matrix [2]. Unlike [2], [3] considers a multiband signal (which is not necessarily WSS), where the spectra at different bands are uncorrelated. This allows [3] to exploit the diagonal structure of the correlation matrix of the entries at different bands.

In wireless communications, the received user signal might suffer from fading and the use of a single receiver to compressively reconstruct either the spectrum or the power spectrum of the user signal might be insufficient to reach the required performance. In order to exploit channel diversity, [4] proposes a cooperative compressive wideband spectrum sensing method for $\mathrm{CR}$ networks, which also reduces the required sampling rate per sensor. However, the aim to reconstruct the spectrum or the spectrum support requires the original spectrum to be sparse. This inspired [5] to extend the power spectrum estimation method of [2] into a cooperative scenario. In [5], the exploitation of the cross-spectra between the compressive measurements at different sensors reduces the required sampling rate per sensor without requiring the power spectrum to be sparse, but it builds upon the knowledge of the channel state information (CSI). As in [5], our work focuses on cooperative compressive power spectrum estimation but we do not need CSI as we do not exploit the cross-spectra between different sensors. We consider groups of sensors where different groups employ different sub-Nyquist sampling patterns. Each group estimates the temporal correlation only for certain lags and not for the entire correlation support. The fusion centre (FC) collects the correlation estimates at different lags produced by different groups of sensors. The combined correlation values at the FC, which should include all the lags in the considered correlation support, are then used to estimate the power spectrum. The required sampling rate per sensor can thus be lower than in the single sensor case presented in [2], while the channel diversity can still be exploited.

Notation: Upper (lower) boldface letters are used to denote matrices (column vectors).

\section{THEORETICAL MODEL}

Consider $Z$ groups of $P$ wireless sensors sensing timedomain WSS signals. At the $(p+1)$-th sensor of the $(z+1)$-th group, we collect $\tilde{N}$ Nyquist-rate samples, split them into $L$ blocks of $N=\tilde{N} / L$ consecutive samples, and collect the samples in the $(l+1)$-th block as $\mathbf{x}_{z, p}[l]=\left[x_{z, p}[l N], x_{z, p}[l N+\right.$ $\left.1], \ldots, x_{z, p}[l N+N-1]\right]^{T}$, with $l=0,1, \ldots, L-1, p=$ $0,1, \ldots, P-1, z=0,1, \ldots, Z-1$, and $x_{z, p}[\tilde{n}]$ the $(\tilde{n}+1)$-th sample. We collect the $(n+1)$-th indices from each block into the set $\{\tilde{n} \in\{0,1, \ldots, \tilde{N}-1\} \mid \tilde{n} \bmod N=n\}$ and label this set as the $(n+1)$-th coset, with the coset index of the $(n+1)$-th coset given by $n$. It is hence possible to view the complete set of $\tilde{N}$ samples as the output of a multi-coset sampler [1] with $N$ cosets and $L$ samples per coset. Next, we introduce compression in each sensor by defining one unique sub-Nyquist sampling pattern for each group. For the $(z+1)$ th group, we define an $M \times N$ selection matrix $\mathbf{C}_{z}$, whose rows are selected from the rows of the $N \times N$ identity matrix $\mathbf{I}_{N}$. The $\frac{M}{N}$-rate compressed version of $\mathbf{x}_{z, p}[l]$ is written as

$$
\mathbf{y}_{z, p}[l]=\mathbf{C}_{z} \mathbf{x}_{z, p}[l], z=0, \ldots, Z-1, p=0, \ldots, P-1 \text {, (1) }
$$

with $\mathbf{y}_{z, p}[l]=\left[y_{z, p}^{(0)}[l], y_{z, p}^{(1)}[l], \ldots, y_{z, p}^{(M-1)}[l]\right]^{T}$. We assume that all $\left\{\mathbf{C}_{z}\right\}_{z=0}^{Z-1}$ in (1) have $M$ rows to simplify the discussion though it is possible for every $\mathbf{C}_{z}$ to have a different number of rows. By writing $\mathbf{C}_{z}$ in (1) in terms of its rows, i.e., $\mathbf{C}_{z}=\left[\mathbf{c}_{z}^{(0)}, \mathbf{c}_{z}^{(1)}, \ldots, \mathbf{c}_{z}^{(M-1)}\right]^{T}$, with $\mathbf{c}_{z}^{(m)}=\left[c_{z}^{(m)}[0]\right.$, $\left.c_{z}^{(m)}[-1], \ldots, c_{z}^{(m)}[1-N]\right]^{T}$, we can write

$$
y_{z, p}^{(m)}[l]=\sum_{n=1-N}^{0} c_{z}^{(m)}[n] x_{z, p}[l N-n],
$$

for $m=0,1, \ldots, M-1$. As (2) can be viewed as a filtering operation of a WSS sequence $x_{z, p}[\tilde{n}]$ by a filter $c_{z}^{(m)}[n]$ followed by an $N$-fold decimation, $\left\{y_{z, p}^{(m)}[l]\right\}_{m=0}^{M-1}$ forms a set of jointly WSS sequences. We then define the set of indices of the $M$ selected cosets in (1) (corresponding to the set of indices of the $M$ rows of $\mathbf{I}_{N}$ used to form $\mathbf{C}_{z}$ ) as

$$
\mathcal{M}_{z}=\left\{n_{z}^{(0)}, n_{z}^{(1)}, \ldots, n_{z}^{(M-1)}\right\}
$$

with $0 \leq n_{z}^{(0)}<n_{z}^{(1)}<\cdots<n_{z}^{(M-1)} \leq N-1$.

We focus on the case where the different sensors observe the same statistics of the received signals, which can be motivated 
by the fact that they are observing the same user signals, that they experience different random fading, and that they use automatic gain control to compensate the difference in the fading variance (path loss and shadowing) in each user band. In this case, we define $r_{x}[\tilde{n}]=E\left[x_{z, p}\left[\tilde{n}^{\prime}\right] x_{z, p}^{*}\left[\tilde{n}^{\prime}-\tilde{n}\right]\right]$, i.e., $r_{x}[\tilde{n}]$ does not vary with sensor indices $p$ or group indices $z$. Note that (3) allows us to write $c_{z}^{(m)}[n]$ in (2) as $c_{z}^{(m)}[n]=\delta\left[n+n_{z}^{(m)}\right]$. As a result, the deterministic cross-correlation between $c_{z}^{(m)}[n]$ and $c_{z}^{\left(m^{\prime}\right)}[n]$, defined as $r_{c_{z}}^{\left(m, m^{\prime}\right)}[n]=\sum_{n^{\prime}=1-N}^{0} c_{z}^{(m)}\left[n^{\prime}\right] c_{z}^{\left(m^{\prime}\right) *}\left[n^{\prime}-n\right]$, is given by

$$
r_{c_{z}}^{\left(m, m^{\prime}\right)}[n]=\delta\left[n+n_{z}^{(m)}-n_{z}^{\left(m^{\prime}\right)}\right]
$$

and the correlations between the measurements at the different cosets $m$ and $m^{\prime}$, i.e., $r_{y_{z}}^{\left(m, m^{\prime}\right)}[l]=E\left[y_{z, p}^{(m)}\left[l^{\prime}\right] y_{z, p}^{\left(m^{\prime}\right) *}\left[l^{\prime}-l\right]\right]$, are related to $r_{x}[\tilde{n}]$ as

$$
\begin{aligned}
r_{y_{z}}^{\left(m, m^{\prime}\right)}[l] & =\sum_{n=1-N}^{N-1} r_{c_{z}}^{\left(m, m^{\prime}\right)}[n] r_{x}[l N-n] \\
& =r_{x}\left[l N+n_{z}^{(m)}-n_{z}^{\left(m^{\prime}\right)}\right] .
\end{aligned}
$$

Remark 1: This paper focuses on estimating $r_{x}[\tilde{n}]$ in (5) at lags $1-N \leq \tilde{n} \leq N-1$ from the sample estimates of $r_{y_{z}}^{\left(m, m^{\prime}\right)}[l]$ in (5) with $L \geq 2$. For this purpose, we can find from (3)-(5) that we need to consider only $r_{y_{z}}^{\left(m, m^{\prime}\right)}[0]$ for all $m$ and $m^{\prime}, r_{y_{z}}^{\left(m, m^{\prime}\right)}[1]$ for all $m<m^{\prime}$, and $r_{y_{z}}^{\left(m, m^{\prime}\right)}[-1]$ for all $m>m^{\prime}$.

Considering (4), we write $\left\{r_{y_{z}}^{\left(m, m^{\prime}\right)}[l]\right\}_{l=-1}^{1}$ in (5) as

$$
\begin{gathered}
r_{y_{z}}^{\left(m, m^{\prime}\right)}[0]=r_{c_{z}}^{\left(m, m^{\prime}\right)}[0] r_{x}[0]+\mathbf{r}_{c_{z}}^{\left(m, m^{\prime}\right) T}[1] \mathbf{r}_{x}[-1] \\
+\mathbf{r}_{c_{z}}^{\left(m, m^{\prime}\right) T}[-1] \mathbf{r}_{x}[1], m, m^{\prime}=0,1, \ldots, M-1, \\
r_{y_{z}}^{\left(m, m^{\prime}\right)}[1]=\mathbf{r}_{c_{z}}^{\left(m, m^{\prime}\right) T}[1] \mathbf{r}_{x}[1], \\
m=0,1, \ldots, M-2, \quad m^{\prime}>m, \\
r_{y_{z}}^{\left(m, m^{\prime}\right)}[-1]=\mathbf{r}_{c_{z}}^{\left(m, m^{\prime}\right) T}[-1] \mathbf{r}_{x}[-1], \\
m^{\prime}=0,1, \ldots, M-2, \quad m>m^{\prime},
\end{gathered}
$$

where we have

$$
\begin{aligned}
\mathbf{r}_{x}[-1] & =\left[r_{x}[1-N], \ldots, r_{x}[-2], r_{x}[-1]\right]^{T}, \\
\mathbf{r}_{x}[1] & =\left[r_{x}[1], r_{x}[2], \ldots, r_{x}[N-1]\right]^{T}, \\
\mathbf{r}_{c_{z}}^{\left(m, m^{\prime}\right)}[-1] & =\left[r_{c_{z}}^{\left(m, m^{\prime}\right)}[-1], \ldots, r_{c_{z}}^{\left(m, m^{\prime}\right)}[1-N]\right]^{T}, \\
\mathbf{r}_{c_{z}}^{\left(m, m^{\prime}\right)}[1] & =\left[r_{c_{z}}^{\left(m, m^{\prime}\right)}[N-1], \ldots, r_{c_{z}}^{\left(m, m^{\prime}\right)}[1]\right]^{T} .
\end{aligned}
$$

Observe from (4), (7c), and (7d) that the first, the second, and the third terms in 6a are non-zero only if $m=$ $m^{\prime}, m<m^{\prime}$, and $m>m^{\prime}$, respectively. Based on this fact, (6b), (6c), and the Hermitian property of $r_{y_{z}}^{\left(m, m^{\prime}\right)}[l]$, i.e., $r_{y_{z}}^{\left(m, m^{\prime}\right)}[l]=r_{y_{z}}^{\left(m^{\prime}, m\right) *}[-l]$, we just need to consider the correlations $r_{y_{z}}^{\left(m, m^{\prime}\right)}$ [0] for $m \geq m^{\prime}$ and $r_{y_{z}}^{\left(m, m^{\prime}\right)}$ [1] for $m^{\prime}>m$, since they contain all relevant information. We then define

$$
\begin{aligned}
\mathbf{r}_{c_{z}}[0]= & {\left[\ldots, r_{c_{z}}^{(m, m)}[0], \ldots\right]^{T}, m=0, \ldots, M-1, } \\
\mathbf{R}_{c_{z}}[-1]=\left[\ldots, \mathbf{r}_{c_{z}}^{\left(m, m^{\prime}\right)}[-1], \ldots\right]^{T}, & \\
& m^{\prime}=0,1, \ldots, M-2, \quad m>m^{\prime}, \\
\mathbf{R}_{c_{z}}[1]= & {\left[\ldots, \mathbf{r}_{c_{z}}^{\left(m, m^{\prime}\right)}[1], \ldots\right]^{T}, } \\
& m=0,1, \ldots, M-2, \quad m^{\prime}>m,
\end{aligned}
$$

with $\mathbf{r}_{c_{z}}[0]=\mathbf{1}_{M}$ (as is clear from (4)) an $M \times 1$ vector having ones in all entries and both $\mathbf{R}_{c_{z}}[1]$ as well as $\mathbf{R}_{c_{z}}[-1]$ $\frac{M(M-1)}{2} \times(N-1)$ matrices. We can then use (8) to write

$$
\begin{aligned}
\mathbf{r}_{y_{z}}^{(0)}[0] & =\mathbf{r}_{c_{z}}[0] r_{x}[0]=\mathbf{1}_{M} r_{x}[0] \\
{\left[\begin{array}{c}
\mathbf{r}_{y_{z}}^{(+)}[0] \\
\mathbf{r}_{y_{z}}^{(-)}[1]
\end{array}\right] } & =\left[\begin{array}{c}
\mathbf{R}_{c_{z}}[-1] \\
\mathbf{R}_{c_{z}}[1]
\end{array}\right] \mathbf{r}_{x}[1]=\mathbf{R}_{c_{z}} \mathbf{r}_{x}[1],
\end{aligned}
$$

with $\mathbf{r}_{y_{z}}^{(0)}[0]=\left[\ldots, r_{y_{z}}^{(m, m)}[0], \ldots\right]^{T}$ for $m=0,1, \ldots, M-1$, $\mathbf{r}_{y_{z}}^{(+)}[0]=\left[\ldots, r_{y z}^{\left(m, m^{\prime}\right)}[0], \ldots\right]^{T}$ for $m^{\prime}=0,1, \ldots, M-2$ and $m>m^{\prime}$, and $\mathbf{r}_{y_{z}}^{(-)}[1]=\left[\ldots, r_{y_{z}}^{\left(m, m^{\prime}\right)}[1], \ldots\right]^{T}$ for $m=$ $0,1, \ldots, M-2$ and $m^{\prime}>m$.

Let us consider an FC collecting the correlation vectors

$$
\mathbf{r}_{y_{z}}=\left[\mathbf{r}_{y_{z}}^{(0) T}[0], \mathbf{r}_{y_{z}}^{(+) T}[0], \mathbf{r}_{y_{z}}^{(-) T}[1]\right]^{T}
$$

from the $(z+1)$-th group of sensors, for $z=0,1, \ldots, Z-$ 1. We collect $\left\{\mathbf{r}_{y_{z}}^{(0)}[0]\right\}_{z=0}^{Z-1}$ in (10) into $\mathbf{r}_{y}^{(0)}[0]=\left[\mathbf{r}_{y_{0}}^{(0) T}[0]\right.$, $\left.\mathbf{r}_{y_{1}}^{(0) T}[0], \ldots, \mathbf{r}_{y_{Z-1}}^{(0) T}[0]\right]^{T}$, similarly define $\mathbf{r}_{y}^{(+)}[0]$ and $\mathbf{r}_{y}^{(-)}[1]$, and use (9) to write

$$
\begin{gathered}
\mathbf{r}_{y}^{(0)}[0]=\mathbf{r}_{c}[0] r_{x}[0]=\mathbf{1}_{M Z} r_{x}[0] \\
{\left[\begin{array}{c}
\mathbf{r}_{y}^{(+)}[0] \\
\mathbf{r}_{y}^{(-)}[1]
\end{array}\right]=\left[\begin{array}{c}
\mathbf{R}_{c}[-1] \\
\mathbf{R}_{c}[1]
\end{array}\right] \mathbf{r}_{x}[1]=\mathbf{R}_{c} \mathbf{r}_{x}[1]}
\end{gathered}
$$

with $\mathbf{r}_{c}[0]=\left[\mathbf{r}_{c_{0}}^{T}[0], \mathbf{r}_{c_{1}}^{T}[0], \ldots, \mathbf{r}_{c_{Z-1}}^{T}[0]\right]^{T}$ and with $\mathbf{R}_{c}[1]$ and $\mathbf{R}_{c}[-1]$ similarly defined as $\mathbf{r}_{c}[0]$. The FC can then use leastsquares (LS) to reconstruct $\mathbf{r}_{x}[1]$ from $\mathbf{r}_{y}^{(+)}[0]$ and $\mathbf{r}_{y}^{(-)}[1]$ if the $\left(M^{2}-M\right) Z \times(N-1)$ matrix $\mathbf{R}_{c}$ in $11 \mathrm{~b}$ has full column rank. Meanwhile, it is clear from (11a) that $r_{x}[0]$ can always be reconstructed using LS and its value is given by the average of the entries of $\mathbf{r}_{y}^{(0)}[0]$. Defining $\mathbf{F}_{2 N-1}$ as the $(2 N-1) \times$ $(2 N-1)$ discrete Fourier transform matrix, the FC can then compute $\mathbf{r}_{x}[-1]$ from $\mathbf{r}_{x}$ [1] using the Hermitian symmetry of $r_{x}[\tilde{n}]$ and the power spectrum from the reconstructed $\mathbf{r}_{x}=$ $\left[r_{x}[0], \mathbf{r}_{x}^{T}[1], \mathbf{r}_{x}^{T}[-1]\right]^{T}$ as

$$
\mathbf{p}_{x}=\mathbf{F}_{2 N-1} \mathbf{r}_{x} .
$$

Due to the finite sensing time, all the correlations must be approximated from a finite number of samples. Consider the unbiased estimate of $r_{y_{z}}^{\left(m, m^{\prime}\right)}[l]$ in (5) given by

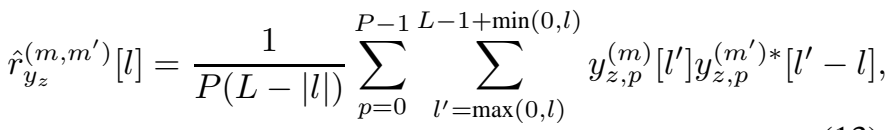

for $m, m^{\prime}=0,1, \ldots, M-1$. Using (13), we then form the estimate of $\mathbf{r}_{y z}$ in 10 as

$$
\hat{\mathbf{r}}_{y_{z}}=\left[\hat{\mathbf{r}}_{y_{z}}^{(0) T}[0], \hat{\mathbf{r}}_{y_{z}}^{(+) T}[0], \hat{\mathbf{r}}_{y_{z}}^{(-) T}[1]\right]^{T} .
$$

Next, we use (14) to form $\hat{\mathbf{r}}_{y}^{(0) T}[0]=\left[\hat{\mathbf{r}}_{y_{0}}^{(0) T}[0], \hat{\mathbf{r}}_{y_{1}}^{(0) T}[0], \ldots\right.$, $\left.\hat{\mathbf{r}}_{y_{Z-1}}^{(0) T}[0]\right]^{T}$ and, similarly also, $\hat{\mathbf{r}}_{y}^{(+)}[0]$ and $\hat{\mathbf{r}}_{y}^{(-)}[1]$, as the estimates of $\mathbf{r}_{y}^{(0)}[0], \mathbf{r}_{y}^{(+)}[0]$, and $\mathbf{r}_{y}^{(-)}[1]$ in (11), respectively. In this case, we apply LS on $\hat{\mathbf{r}}_{y}^{(0)}[0], \hat{\mathbf{r}}_{y}^{(+)}[0]$, and $\hat{\mathbf{r}}_{y}^{(-)}[1]$ instead of $\mathbf{r}_{y}^{(0)}[0], \mathbf{r}_{y}^{(+)}[0]$, and $\mathbf{r}_{y}^{(-)}[1]$ in (11).

\section{CONDITION FOR LEAST-SQUARES RECONSTRUCTION}

We now focus on the LS reconstruction of $\mathbf{r}_{x}[1]$ in $111 \mathrm{~b}$ and first evaluate the full column rank condition of $\mathbf{R}_{c}$ for $Z=1$. Note from (9b) and (11b) that $\mathbf{R}_{c}=\mathbf{R}_{c_{z}}$ for $Z=1$. 
We can find from (4), (7c), (7d), and (8) that each row of $\mathbf{R}_{c_{z}}$ in $(9 b)$ has only a single one in one entry and zeros elsewhere. The full column rank condition of $\mathbf{R}_{c_{z}}$ is then ensured if and only if each of its columns has at least a single one. We define $\Omega\left(\mathcal{M}_{z}\right)=\left\{\left(n_{z}^{(m)}-n_{z}^{\left(m^{\prime}\right)}\right) \bmod N \mid \forall n_{z}^{(m)}, n_{z}^{\left(m^{\prime}\right)} \in \mathcal{M}_{z}\right\}$ and review the definition of a circular sparse ruler in [6], which is also known as a cyclic difference set [7].

Definition 1: A length- $(N-1)$ circular sparse ruler is a set $\mathcal{Q} \subset\{0,1, \ldots, N-1\}$ such that $\Omega(\mathcal{Q})=\{0,1, \ldots, N-1\}$. A length- $(N-1)$ circular sparse ruler is a ruler that can measure all integers from 0 to $N-1$ in a modular fashion despite missing some of its integer marks. Let us consider the following theorem.

Theorem 1: $\mathbf{R}_{c_{z}}$ in (9b) has full column rank if and only if $\mathcal{M}_{z}$ in (3) is a circular sparse ruler of length $N-1$. Proof: Assuming $n_{z}^{(m)}>n_{z}^{\left(m^{\prime}\right)}$, having a one in the $\left(n_{z}^{(m)}-\right.$ $\left.n_{z}^{\left(m^{\prime}\right)}\right)$-th entry of $\mathbf{r}_{c_{z}}^{\left(m, m^{\prime}\right)}[-1]$ in (7c) implies having a one in the $\left(n_{z}^{\left(m^{\prime}\right)}-n_{z}^{(m)}+N\right)$-th entry of $\mathbf{r}_{c_{z}}^{\left(m^{\prime}, m\right)}$ [1] in (7d). It is then clear from (8) that if $\mathbf{R}_{c_{z}}$ in $9 \mathrm{~b}$ ) has at least a single one in the $\left(\left(n_{z}^{(m)}-n_{z}^{\left(m^{\prime}\right)}\right) \bmod N\right)$-th column, it also has at least a single one in the $\left(\left(n_{z}^{\left(m^{\prime}\right)}-n_{z}^{(m)}\right) \bmod N\right)$-th column. As we need to ensure each column of $\mathbf{R}_{c_{z}}$ to have at least a single one, ensuring $\mathbf{R}_{c_{z}}$ in $9 \mathrm{~b}$ to have full column rank is identical to ensuring that $\Omega\left(\mathcal{M}_{z}\right)=\{0,1, \ldots, N-1\}$. By considering Definition 1 , the proof is concluded.

The correlation reconstruction with $Z=1$ is equivalent to the work in [2], though [2] does not directly relate the rank condition of the system matrix to a circular sparse ruler. For $Z>1$, observe from (11b) that $\mathbf{R}_{c_{z}}$ in $9 \mathrm{~b}$ does not need to have full column rank and thus each of the sets $\left\{\mathcal{M}_{z}\right\}_{z=0}^{Z-1}$ does not have to be a circular sparse ruler. In fact, for $Z>1$, $\mathbf{R}_{c}$ in $11 \mathrm{~b}$ has full column rank if

$$
\bigcup_{z=0}^{Z-1} \Omega\left(\mathcal{M}_{z}\right)=\{0,1, \ldots, N-1\} \text {. }
$$

For completeness, let us consider the following definition.

Definition 2: A length- $(N-1)$ incomplete circular sparse ruler is a set $\mathcal{Q} \subset\{0,1, \ldots, N-1\}$ such that $\Omega(\mathcal{Q}) \subsetneq$ $\{0,1, \ldots, N-1\}$.

Like a length- $(N-1)$ circular sparse ruler, a length- $(N-1)$ incomplete circular sparse ruler misses some of its integer marks, but it cannot measure all integers from 0 to $N-1$ in a modular fashion. Using Definition 2, we can view the design of $\left\{\mathcal{M}_{z}\right\}_{z=0}^{Z-1}$ satisfying (15) as having $Z$ circular rulers, preferably sparse and incomplete to achieve a strong compression, such that, for each $n \in\{0,1, \ldots, N-1\}$, at least one of these $Z$ incomplete circular sparse rulers can measure the distance $n$ in a modular fashion. Clearly, there is a tradeoff between $Z$ and $M$ under the constraint of 15 since it is not possible to minimize both of them. Here, we aim at minimizing $Z$ given $M$, i.e.,

$$
\min _{Z,\left\{\mathcal{M}_{z}\right\}_{z=0}^{Z-1}} Z \text { s.t. } 15 \text { and }\left|\mathcal{M}_{z}\right|=M, \forall z \text {. }
$$

Note that maximizing $\left|\Omega\left(\mathcal{M}_{z}\right)\right|$ in (15) for all $z$ implies minimizing $Z$. As we have $\left(\left(n_{z}^{\left(m^{\prime}\right)}-n_{z}^{(m)}\right) \bmod N\right) \in \Omega\left(\mathcal{M}_{z}\right)$ and $\left(\left(n_{z}^{(m)}-n_{z}^{\left(m^{\prime}\right)}\right) \bmod N\right) \in \Omega\left(\mathcal{M}_{z}\right)$ whenever $n_{z}^{(m)}, n_{z}^{\left(m^{\prime}\right)} \in$ $\mathcal{M}_{z}$, we can find that $\left|\Omega\left(\mathcal{M}_{z}\right)\right| \leq M(M-1)+1$. Disregarding the self-difference of the coset indices, we can find from (15) that $Z$ is bounded as $Z \geq\left\lceil\frac{N-1}{M(M-1)}\right]$. We thus aim to have $\left|\Omega\left(\mathcal{M}_{z}\right)\right|$ as close as possible to $M(M-1)+1$ for all $z$.

Definition 3: A length- $(N-1)$ circular Golomb ruler is a set $\mathcal{Q} \subset\{0,1, \ldots, N-1\}$ such that, if $q, q^{\prime}, \tilde{q}, \tilde{q}^{\prime} \in \mathcal{Q}$ with $q \neq q^{\prime}$, then $\left(q-q^{\prime}\right) \bmod N=\left(\tilde{q}-\tilde{q}^{\prime}\right) \bmod N$ implies $q=\tilde{q}$ and $q^{\prime}=\tilde{q}^{\prime}[8]$. We say that $Z$ length- $(N-1)$ circular Golomb rulers $\left\{\mathcal{Q}_{z}\right\}_{z=0}^{Z-1}$ are non-overlapping if $\bigcap_{z=0}^{Z-1} \Omega\left(\mathcal{Q}_{z}\right)=\{0\}$.

Definition 3 implies that $\left|\Omega\left(\mathcal{M}_{z}\right)\right|=M(M-1)+1$ if and only if $\mathcal{M}_{z}$ is a circular Golomb ruler. Hence, a way to (approximately) minimize $Z$ given $M$ is to search for nonoverlapping circular Golomb rulers that cover all or a certain number of the $N$ integer distances. In the latter case, we continue to search for another circular Golomb or an ordinary incomplete circular sparse ruler with $M$ marks that covers as many of the remaining uncovered integer distances as possible. This step is repeated until all the $N$ integer distances are covered. For $M=2$, the lower bound for $Z$ is $\left\lceil\frac{N-1}{2}\right\rceil$, which is reached for $N$ odd by having $\frac{N-1}{2}$ non-overlapping circular Golomb rulers with the $(z+1)$-th ruler $\mathcal{M}_{z}=\{0, z+1\}$. This bound is also reached for $N$ even by having $\frac{N}{2}-1$ nonoverlapping circular Golomb rulers with $\mathcal{M}_{z}=\{0, z+1\}$, plus one incomplete circular sparse ruler $\mathcal{M}_{\frac{N}{2}-1}=\left\{0, \frac{N}{2}\right\}$ as the last ruler. For $M=3$, the lower bound for $Z$ is $\left\lceil\frac{N-1}{6}\right\rceil$. Table I provides a list of non-overlapping circular Golomb rulers that achieve this bound for integers $\frac{N-1}{6}$ and $43 \leq N \leq 115$. These non-overlapping circular Golomb rulers are found by first having $\mathcal{M}_{z}=\{0, z+1\}$, for all $z=0,1, \ldots, Z-1$. The third (last) coset index in $\mathcal{M}_{z}$, for each $z$, is then determined by using a greedy search from the remaining coset indices that have not yet been used in $\left\{\mathcal{M}_{z}\right\}_{z=0}^{Z-1}$. We are still investigating if there is always a sampling pattern that reaches the lower bound for $Z$, for any value of $N$ and $M$, and if there is a better algorithm to find this optimal sampling pattern. A set of non-overlapping linear (instead of circular) Golomb rulers called a perfect difference basis system is discussed in [9].

\section{NUMERICAL StUdY}

We consider $N=103$ and six user signals whose frequency bands are given in Table $\Pi$ together with the power at each band normalized by frequency. These signals are generated by passing six sets of circular complex zero-mean Gaussian i.i.d. noise signals, with the variances set according to the desired user signal powers, into different digital filters having $N$ taps where the location of the unit-gain passband of the filter for each realization corresponds to the six different active bands. We assume that the signals are observed by unsynchronized sensors, which means that, at a certain point in time, all sensors generally observe different parts of the user signals. To simplify the experiment, we assume that, at time $t$, the $(z P+p)$-th sensor observes the part of the user signals that has previously been observed by the $(z P+p-1)$-th sensor at time $t-14 T$, with $T$ the Nyquist sampling time. We start from $M=3$ active cosets per sensor and fix $Z$ at its lower bound, which is $Z=17$ for $M=3$, by having 17 non-overlapping circular Golomb rulers (search for $N=103$ in Table II). We 
set the white noise power at each sensor to $16 \mathrm{dBm}$ and vary $M, P$ and $L$ (see Fig. 1). Each user signal received by different sensors is assumed to pass through different wireless channels but the signal from a user received by all sensors is assumed to experience the same path loss and shadowing. Table Iindicates the amount of path loss experienced between each user and all sensors, which is assumed to include shadowing. We simulate the Rayleigh fading by generating the channel frequency response based on a zero-mean complex Gaussian distribution with variance governed by the path loss in Table I] Each band is assumed to experience flat fading. We compute the normalized mean square error (NMSE) of the compressively estimated power spectrum with respect to the Nyquist-rate based estimate (obtained by activating all $N$ cosets in each sensor). Fig. 1 shows how increasing the compression rate per sensor up to $M / N=0.19$ improves the estimation quality. The estimation quality is also improved by having either more sensors $P Z$ or more samples per coset $L$.

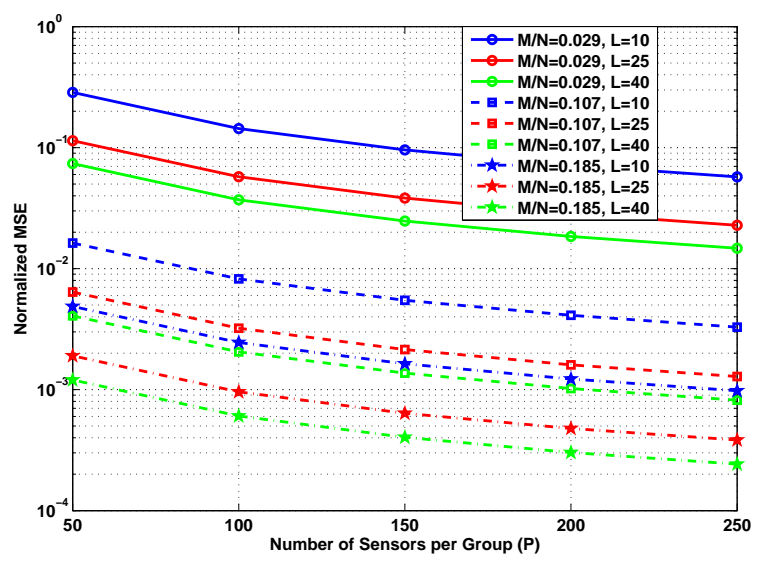

Fig. 1. The NMSE between the compressively reconstructed power spectrum and the one reconstructed from the Nyquist rate samples.

TABLE I

THE FREQUENCY BANDS OCCUPIED BY THE USERS, THEIR POWER, AND THE EXPERIENCED PATH LOSS

\begin{tabular}{|c|c|c|}
\hline User band (rad/sample) & Power/freq. (per rad/sample) & Path loss \\
\hline$\left[\frac{-8 \pi}{9}, \frac{-7 \pi}{9}\right]$ & $38 \mathrm{dBm}$ & $-18 \mathrm{~dB}$ \\
\hline$\left[\frac{-6 \pi}{9}, \frac{-5 \pi}{9}\right]$ & $40 \mathrm{dBm}$ & $-19 \mathrm{~dB}$ \\
\hline$\left[\frac{\pi}{9}, \frac{2 \pi}{9}\right]$ & $34 \mathrm{dBm}$ & $-11 \mathrm{~dB}$ \\
\hline$\left[\frac{3 \pi}{9}, \frac{4 \pi}{9}\right]$ & $34 \mathrm{dBm}$ & $-17 \mathrm{~dB}$ \\
\hline$\left[\frac{4 \pi}{9}, \frac{5 \pi}{9}\right]$ & $32 \mathrm{dBm}$ & $-13 \mathrm{~dB}$ \\
\hline$\left[\frac{6 \pi}{9}, \frac{7 \pi}{9}\right]$ & $35 \mathrm{dBm}$ & $-19 \mathrm{~dB}$ \\
\hline
\end{tabular}

\section{REFERENCES}

[1] M. Mishali and Y.C. Eldar, "Blind multiband signal reconstruction: compressed sensing for analog signals," IEEE Trans. Signal Process. vol. 57, no. 3, pp. 993-1009, March 2009.

[2] D.D. Ariananda and G. Leus, "Compressive wideband power spectrum estimation,” IEEE Trans. Signal Process., vol. 60, no. 9, pp. 4775-4789, Sep. 2012.

[3] C.P. Yen, Y. Tsai, and X. Wang, "Wideband spectrum sensing based on sub-Nyquist sampling," IEEE Trans. Signal Process., vol. 61, no. 12, pp. 3028-3040, June 2013.

[4] F. Zeng, C. Li, and Z. Tian, "Distributed compressive spectrum sensing in cooperative multihop cognitive networks," IEEE J. Sel. Topics Signal Process., vol. 5, no.1, pp. 37-48, February 2011.

[5] D.D. Ariananda and G. Leus, "Cooperative compressive wideband power spectrum sensing," Proc. Asilomar Conference on Signals, Systems and Computers, Pacific Grove, pp. 303-307, November 2012.
[6] D. Romero and G. Leus, "Compressive covariance sampling," Proc. Inf. Theory Appl. Workshop (ITA 2013), San Diego, California, Feb. 2013.

[7] J.C.P. Miller, "Difference bases: three problems in additive number theory," Comput. in Number Theory, pp. 299-322, Acad. Press, 1971.

[8] C.J. Colbourn and J.H. Dinitz, Handbook of combinatorial designs, Chapman and Hall/CRC, Boca Raton, Florida, 2007.

[9] P. Wild, "Difference basis systems," Discrete Mathematics, vol. 63, pp. 81-90, 1987.

TABLE II

LIST OF NON-OVERLAPPING CIRCULAR GOLOMB RULERS THAT COVER ALL OF THE $N$ INTEGER DISTANCES

\begin{tabular}{|c|c|}
\hline $\bar{N}$ & Non-overlapping circular Golomb rulers \\
\hline 43 & $\begin{array}{l}\mathcal{M}_{0}=\{0,1,17\}, \mathcal{M}_{1}=\{0,2,12\}, \mathcal{M}_{2}=\{0,3,24\}, \\
\mathcal{M}_{3}=\{0,4,13\}, \mathcal{M}_{4}=\{0,5,28\}, \mathcal{M}_{5}=\{0,6,14\}, \\
\mathcal{M}_{6}=\{0,7,32\}\end{array}$ \\
\hline$\overline{49}$ & $\begin{array}{l}\mathcal{M}_{0}=\{0,1,13\}, \mathcal{M}_{1}=\{0,2,20\}, \mathcal{M}_{2}=\{0,3,14\}, \\
\mathcal{M}_{3}=\{0,4,30\}, \mathcal{M}_{4}=\{0,5,15\}, \mathcal{M}_{5}=\{0,6,27\}, \\
\mathcal{M}_{6}=\{0,7,16\}, \mathcal{M}_{7}=\{0,8,25\}\end{array}$ \\
\hline 55 & $\begin{array}{l}\mathcal{M}_{0}=\{0,1,15\}, \mathcal{M}_{1}=\{0,2,23\}, \mathcal{M}_{2}=\{0,3,16\}, \\
\mathcal{M}_{3}=\{0,4,33\}, \mathcal{M}_{4}=\{0,5,17\}, \mathcal{M}_{5}=\{0,6,31\}, \\
\mathcal{M}_{6}=\{0,7,18\}, \mathcal{M}_{7}=\{0,8,28\}, \mathcal{M}_{8}=\{0,9,19\}\end{array}$ \\
\hline 61 & $\begin{array}{l}\mathcal{M}_{0}=\{0,1,16\}, \mathcal{M}_{1}=\{0,2,39\}, \mathcal{M}_{2}=\{0,3,17\}, \\
\mathcal{M}_{3}=\{0,4,38\}, \mathcal{M}_{4}=\{0,5,18\}, \mathcal{M}_{5}=\{0,6,35\}, \\
\mathcal{M}_{6}=\{0,7,19\}, \mathcal{M}_{7}=\{0,8,36\}, \mathcal{M}_{8}=\{0,9,20\}, \\
\mathcal{M}_{9}=\{0,10,31\}\end{array}$ \\
\hline 67 & $\begin{array}{l}\mathcal{M}_{0}=\{0,1,18\}, \mathcal{M}_{1}=\{0,2,42\}, \mathcal{M}_{2}=\{0,3,19\}, \\
\mathcal{M}_{3}=\{0,4,41\}, \mathcal{M}_{4}=\{0,5,20\}, \mathcal{M}_{5}=\{0,6,35\}, \\
\mathcal{M}_{6}=\{0,7,21\}, \mathcal{M}_{7}=\{0,8,36\}, \mathcal{M}_{8}=\{0,9,22\}, \\
\mathcal{M}_{9}=\{0,10,34\}, \mathcal{M}_{10}=\{0,11,23\}\end{array}$ \\
\hline 73 & $\begin{array}{l}\mathcal{M}_{0}=\{0,1,43\}, \mathcal{M}_{1}=\{0,2,20\}, \mathcal{M}_{2}=\{0,3,44\} \\
\mathcal{M}_{3}=\{0,4,21\}, \mathcal{M}_{4}=\{0,5,40\}, \mathcal{M}_{5}=\{0,6,22\} \\
\mathcal{M}_{6}=\{0,7,34\}, \mathcal{M}_{7}=\{0,8,23\}, \mathcal{M}_{8}=\{0,9,28\} \\
\mathcal{M}_{9}=\{0,10,24\}, \mathcal{M}_{10}=\{0,11,37\}, \mathcal{M}_{11}=\{0,12,25\}\end{array}$ \\
\hline 79 & $\begin{array}{l}\mathcal{M}_{0}=\{0,1,28\}, \mathcal{M}_{1}=\{0,2,21\}, \mathcal{M}_{2}=\{0,3,48\}, \\
\mathcal{M}_{3}=\{0,4,22\}, \mathcal{M}_{4}=\{0,5,40\}, \mathcal{M}_{5}=\{0,6,23\}, \\
\mathcal{M}_{6}=\{0,7,49\}, \mathcal{M}_{7}=\{0,8,24\}, \mathcal{M}_{8}=\{0,9,50\}, \\
\mathcal{M}_{9}=\{0,10,25\}, \mathcal{M}_{10}=\{0,11,47\}, \mathcal{M}_{11}=\{0,12,26\} \\
\mathcal{M}_{12}=\{0,13,33\}\end{array}$ \\
\hline 85 & $\begin{array}{l}\mathcal{M}_{0}=\{0,1,32\}, \mathcal{M}_{1}=\{0,2,23\}, \mathcal{M}_{2}=\{0,3,50\} \\
\mathcal{M}_{3}=\{0,4,24\}, \mathcal{M}_{4}=\{0,5,51\}, \mathcal{M}_{5}=\{0,6,25\} \\
\mathcal{M}_{6}=\{0,7,48\}, \mathcal{M}_{7}=\{0,8,26\}, \mathcal{M}_{8}=\{0,9,49\} \\
\mathcal{M}_{9}=\{0,10,27\}, \mathcal{M}_{10}=\{0,11,33\}, \mathcal{M}_{11}=\{0,12,28\} \\
\mathcal{M}_{12}=\{0,13,43\}, \mathcal{M}_{13}=\{0,14,29\}\end{array}$ \\
\hline 91 & $\begin{array}{l}\mathcal{M}_{0}=\{0,1,59\}, \mathcal{M}_{1}=\{0,2,24\}, \mathcal{M}_{2}=\{0,3,54\} \\
\mathcal{M}_{3}=\{0,4,25\}, \mathcal{M}_{4}=\{0,5,41\}, \mathcal{M}_{5}=\{0,6,26\}, \\
\mathcal{M}_{6}=\{0,7,56\}, \mathcal{M}_{7}=\{0,8,27\}, \mathcal{M}_{8}=\{0,9,52\}, \\
\mathcal{M}_{9}=\{0,10,28\}, \mathcal{M}_{10}=\{0,11,57\}, \mathcal{M}_{11}=\{0,12,29\}, \\
\mathcal{M}_{12}=\{0,13,60\}, \mathcal{M}_{13}=\{0,14,30\}, \mathcal{M}_{14}=\{0,15,38\}\end{array}$ \\
\hline 97 & $\begin{array}{l}\mathcal{M}_{0}=\{0,1,18\}, \mathcal{M}_{1}=\{0,2,21\}, \mathcal{M}_{2}=\{0,3,23\}, \\
\mathcal{M}_{3}=\{0,4,26\}, \mathcal{M}_{4}=\{0,5,29\}, \mathcal{M}_{5}=\{0,6,31\}, \\
\mathcal{M}_{6}=\{0,7,34\}, \mathcal{M}_{7}=\{0,8,36\}, \mathcal{M}_{8}=\{0,9,65\}, \\
\mathcal{M}_{9}=\{0,10,60\}, \mathcal{M}_{10}=\{0,11,46\}, \mathcal{M}_{11}=\{0,12,67\}, \\
\mathcal{M}_{12}=\{0,13,52\}, \mathcal{M}_{13}=\{0,14,54\}, \mathcal{M}_{14}=\{0,15,59\}, \\
\mathcal{M}_{15}=\{0,16,64\}\end{array}$ \\
\hline 103 & $\begin{array}{l}\mathcal{M}_{0}=\{0,1,27\}, \mathcal{M}_{1}=\{0,2,39\}, \mathcal{M}_{2}=\{0,3,28\}, \\
\mathcal{M}_{3}=\{0,4,62\}, \mathcal{M}_{4}=\{0,5,29\}, \mathcal{M}_{5}=\{0,6,65\}, \\
\mathcal{M}_{6}=\{0,7,30\}, \mathcal{M}_{7}=\{0,8,57\}, \mathcal{M}_{8}=\{0,9,31\}, \\
\mathcal{M}_{9}=\{0,10,50\}, \mathcal{M}_{10}=\{0,11,32\}, \mathcal{M}_{11}=\{0,12,60\}, \\
\mathcal{M}_{12}=\{0,13,33\}, \mathcal{M}_{13}=\{0,14,56\}, \mathcal{M}_{14}=\{0,15,34\}, \\
\mathcal{M}_{15}=\{0,16,52\}, \mathcal{M}_{16}=\{0,17,35\}\end{array}$ \\
\hline 109 & $\begin{array}{l}\mathcal{M}_{0}=\{0,1,28\}, \mathcal{M}_{1}=\{0,2,42\}, \mathcal{M}_{2}=\{0,3,29\}, \\
\mathcal{M}_{3}=\{0,4,61\}, \mathcal{M}_{4}=\{0,5,30\}, \mathcal{M}_{5}=\{0,6,70\}, \\
\mathcal{M}_{6}=\{0,7,31\}, \mathcal{M}_{7}=\{0,8,68\}, \mathcal{M}_{8}=\{0,9,32\}, \\
\mathcal{M}_{9}=\{0,10,66\}, \mathcal{M}_{10}=\{0,11,33\}, \mathcal{M}_{11}=\{0,12,71\}, \\
\mathcal{M}_{12}=\{0,13,34\}, \mathcal{M}_{13}=\{0,14,58\}, \mathcal{M}_{14}=\{0,15,35\}, \\
\mathcal{M}_{15}=\{0,16,63\}, \mathcal{M}_{16}=\{0,17,36\}, \mathcal{M}_{17}=\{0,18,55\}\end{array}$ \\
\hline 115 & $\begin{array}{l}\mathcal{M}_{0}=\{0,1,30\}, \mathcal{M}_{1}=\{0,2,45\}, \mathcal{M}_{2}=\{0,3,31\}, \\
\mathcal{M}_{3}=\{0,4,64\}, \mathcal{M}_{4}=\{0,5,32\}, \mathcal{M}_{5}=\{0,6,73\}, \\
\mathcal{M}_{6}=\{0,7,33\}, \mathcal{M}_{7}=\{0,8,71\}, \mathcal{M}_{8}=\{0,9,34\}, \\
\mathcal{M}_{9}=\{0,10,69\}, \mathcal{M}_{10}=\{0,11,35\}, \mathcal{M}_{11}=\{0,12,74\}, \\
\mathcal{M}_{12}=\{0,13,36\}, \mathcal{M}_{13}=\{0,14,61\}, \mathcal{M}_{14}=\{0,15,37\}, \\
\mathcal{M}_{15}=\{0,16,66\}, \mathcal{M}_{16}=\{0,17,38\}, \mathcal{M}_{17}=\{0,18,58\}, \\
\mathcal{M}_{18}=\{0,19,39\}\end{array}$ \\
\hline
\end{tabular}

